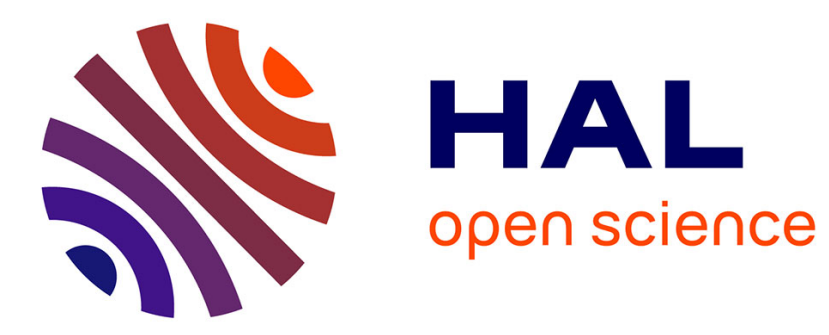

\title{
Playing with senses in VR: Alternate perceptions combining vision and touch
}

\author{
Anatole Lécuyer
}

\section{To cite this version:}

Anatole Lécuyer. Playing with senses in VR: Alternate perceptions combining vision and touch. IEEE

Computer Graphics and Applications, 2017, 37 (1), pp.20-26. 10.1109/MCG.2017.14 . hal-01848314

\section{HAL Id: hal-01848314 \\ https://hal.science/hal-01848314}

Submitted on 24 Jul 2018

HAL is a multi-disciplinary open access archive for the deposit and dissemination of scientific research documents, whether they are published or not. The documents may come from teaching and research institutions in France or abroad, or from public or private research centers.
L'archive ouverte pluridisciplinaire HAL, est destinée au dépôt et à la diffusion de documents scientifiques de niveau recherche, publiés ou non, émanant des établissements d'enseignement et de recherche français ou étrangers, des laboratoires publics ou privés. 


\title{
Playing with senses in VR: Alternate perceptions combining vision and touch
}

\author{
Anatole Lécuyer, Inria, Rennes, France \\ anatole.lecuyer@inria.fr
}

\begin{abstract}
Virtual Reality is an immersive experience based on computer-generated stimulations perceived with multiple sensory channels. It is possible to manipulate these sensory stimulations independently and create conflicting situations in which, for instance, vision and touch are spatially and/or temporally inconsistent. In this article we show how to exploit these ambiguous sensorial situations in order to generate new kinds of percept but also plausible $3 D$ interactions in virtual environments. We particularly insist on three results obtained by playing with visual and haptic senses in virtual reality: (i) pseudo-haptic effects, (ii) self-motion sensations, and (iii) bodyownership illusions.
\end{abstract}

\section{Conflicts and coherence of senses in virtual reality}

Virtual Reality (VR) technologies aim at generating the sensory illusion of an alternate reality: being located in a different place, or interacting with objects or characters that are not physically present in the real surrounding of the user. A sensory illusion is a matter of interpretation. Our senses send messages that are consistent with the stimulations they receive, but the resulting combination of them is somehow inconsistent, and the brain is fooled in its interpretation and final percept (Berthoz, 2002).

In this quest for creating a proper sensory illusion, VR settings put the user in a situation of psychological conflict between two situations and two living experiences: the real situation (in the real setup) and the artificial one (in the virtual environment). In general, the real and the virtual situations share common properties, such as the same shape of floor, so that the fusion or transition between both situations is smoother. The real setup remains mostly stable in time, and everything is made to make it imperceptible - which is literally the case when putting on a head-mounted display (HMD). So-called "breaks in presence" can occur whenever a discrepancy between the virtual and the real situations is noticed or when the virtual stimulation becomes less reliable, e.g., with a high latency (Slater, 2000).

If "it works", and when this psychological conflict is solved in favor of the virtual situation, the resulting sensory experience and subsequent feeling of immersion can be very strong. This can be revealed by physiological or behavioral reactions of users. A good example is the vertigo sensation felt when positioned at the top of a virtual pit and looking down. People would often refuse to jump into the virtual void, even though they know they are safely standing on a flat floor in reality. Interestingly, when a real physical edge is added on the floor, for instance using a wooden plate, the physiological reaction is increasing significantly compare to the situation with no physical edge (Meehan, 2002). Thus, the vertigo sensation is stronger in presence of an additional and consistent tactile cue. The immersion feeling is higher in presence of a sensory redundancy in VR.

An immersive experience relies on realistic sensory stimulations, essentially visual, but sometimes audio or tactile (haptic). In practice, it is often impossible to perfectly reproduce a multi-sensory experience in VR with all the sensory stimulations involved. In a paradoxical manner, the VR experience is usually a situation of "sensory deprivation": a perceptual isolation with multiple senses being removed or cut off. Incidentally, in terms of interaction capabilities, the user is mostly in situation of handicap, being unable to achieve basic operations, provided with limited possibilities of perception and action. 

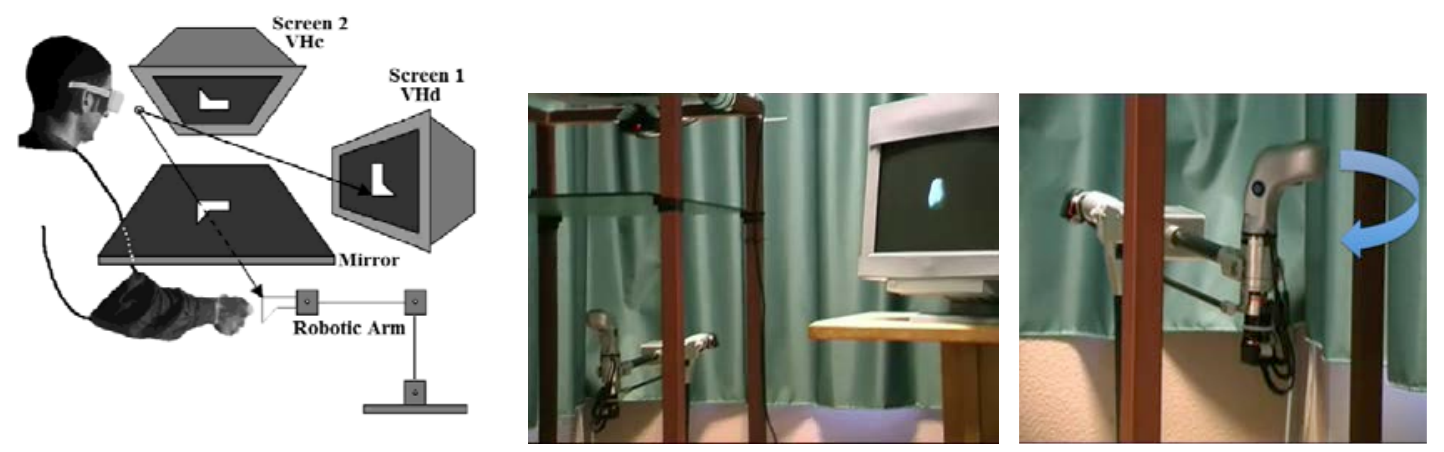

Figure 1: What happens when vision and touch are not collocated ? In our experiment (Congedo, 2006), participants could watch and grasp a rotating virtual handle under two different conditions: VHc (Visual and Haptic information are spatially "Collocated" - as in HMD-based VR settings) and VHd (Vision and Haptics are "De-located", as in screen-based VR settings).

Sensory redundancy is an effective means for gaining a higher immersion. But the sensory stimulations are expected to remain consistent spatially and temporally. A spatial or temporal discrepancy between sensory sources is expected to decrease the plausibility of the virtual experience. In (Congedo, 2006), we could show that when visual and haptic information are not spatially colocated, the multi-sensory integration is negatively impacted, and the weight given to the haptic modality decreases strongly in favor of vision (see Figure 1). The spatial offset between the visual and haptic displays ends up with a masking of the haptic sensation. Interestingly, designers of VR systems can relate these findings to the relevance of the two sensory channels. For instance, if the contribution of touch is important for the task, great efforts should be undertaken to collocate as much as possible the visual and haptic percepts. On the other hand, in presence of a low-quality haptic feedback, the interest may be to contain the limitations of the haptic device by keeping distant the two displays.

Virtual Reality can be used to create experimental situations that can sometimes hardly be reproduced in a real setup such as artificial sensory conflicts. A sensory conflict implies that the information coming from one modality differs from the information coming from another one. Sensory conflicts can be a source of problems in VR. According to the sensory conflict theory, "cybersickness" is evoked as the result of an inconsistency between the visual and the vestibular or proprioceptive senses. But sensory conflicts can also help psychologists to better understand how humans perceive multisensory information, enabling for instance to compute the relative weights attributed to the various sensory channels (Ernst, 2002). For instance, when spatial interaction tasks are concerned, visuohaptic perception was found to be characterized by a strong visual dominance (Rock, 1964). In this context the concept of sensory coherence is also central when perceiving and representing the environment with multiple senses. In this perspective, sensory signals are not processed to directly estimate the relevant variables, but rather to estimate the difference between mental estimations and the relevant variables (Cornilleau-Pérès, 1993).

In this article, we will show how sensory conflicts can become a source of inspiration for VR designers and, more generally, how combinations of visual and haptic feedbacks can be exploited to generate alternate percepts and novel 3D interaction schemes. We will insist on three series of results obtained recently on this research topic: pseudo-haptic effects, self-motion sensations, and bodyownership illusions. All of them were obtained by playing with redundant or conflicting visual and haptic cues in virtual environments, bordering systematically on sensory illusions.

\section{Haptic illusions and "pseudo-haptic feedback"}

As a first example, "pseudo-haptic feedback" intends to produce a wide range of haptic sensations, such as friction, relief or stiffness, without using a haptic interface but playing with visual feedback. 
We state four key assertions concerning pseudo-haptic feedback (Lécuyer, 2009). First, pseudo-haptic feedback implies one or more sensory conflicts between visual and haptic cues. Second, pseudo-haptic feedback relies on the sensory dominance of vision over touch when perceiving spatial properties (distance, position, size, displacement, etc). Third, pseudo-haptic feedback corresponds to a new and coherent representation of the environment resulting from a combination of haptic and visual information. Fourth, pseudo-haptic feedback can create a haptic illusion, i.e., the perception of a haptic property different from the one present in the real environment.

Since our first article published on this topic in 2000 (Lécuyer, 2009), we have designed and studied numerous examples of pseudo-haptic effects. We provide hereafter a representative set of successful studies and setups.

The most famous technique based on pseudo-haptic feedback has been originally designed in a 2D context: the "pseudo-haptic textures" (Lécuyer, 2009). This pseudo-haptic effect was meant to display the relief of 2D images using a simple computer mouse. When the user manipulates the mouse, the technique consists of altering the cursor's visual motion as it moves over the image, i.e. manipulating the Control/Display ratio. To create the impression that the cursor is climbing up a slope, it is slowed down. Inversely, to simulate the cursor sliding down a slope, it is speeded up. For example, to simulate the cursor moving over a bump (as illustrated in Figure 2A), the cursor is slowed down until it reaches the top of the bump. Once it is past the top, the cursor accelerates, until it reaches the foot of the bump. After that, it returns to its normal speed. This technique has been evaluated within an extensive series of experiments that demonstrated that participants were able to well recognize and precisely draw texture patterns simulated using pseudo-haptic textures. Later on, we proposed an extension of this technique called "Elastic Images" aiming at simulating the local elasticity of images (Argelaguet, 2013). The elasticity sensation is generated by a procedural image deformation algorithm that modifies the image according to its simulated physical properties and to the virtual pressure exerted by the user (see Figure 2C). The simulated pressure depends on the time the user keeps the mouse button pressed. A psychophysical experiment showed that users were able to recognize up to eight different elasticity configurations.

More recently pseudo-haptic feedback has been studied in the context of 3D interaction with virtual environments for improving the selection or manipulation of virtual objects. One example illustrated in Figure 2B improves the selection of items enclosed in a 3D carousel designed for virtual showcasing (Gaucher, 2013). The carousel is a 3D ring menu rendered on a 3D display. The 3D interaction with the carousel is achieved by tracking user's gestures: in order to rotate the carousel, the user has to perform swipe gestures. Our pseudo-haptic effect is introduced to highlight relevant items, such as promotional products, by locally modifying the friction of the carousel. This effect is expected to "attract" the user towards these specific items when interacting with the carousel. When facing an item with a strong friction coefficient, the user must increase the amplitude of movement of the hand to move to the following or previous item. A second example is a unique interaction paradigm called the "Virtual Mitten" (Achibet, 2014). It is meant to simulate 3D manipulation of objects using grip forces. It is based on the passive haptic feedback provided by a handheld elastic input device (an engineered hand-exerciser), and the visual metaphor of a mitten that enables to grasp and manipulate 3D objects (see Figure 2E). The grip force exerted on the device enables to grasp objects and achieve various manipulation tasks, such as opening a drawer or pulling a lever. The grasping performed by the virtual mitten is directly correlated with the grip force applied on the elastic device. A pseudohaptic effect is then introduced in order to generate the haptic perception of different levels of grasping effort. A psychophysical experiment could show that that participants were well able to perceive different levels of effort during several manipulation tasks thanks to this pseudo-haptic approach.

Pseudo-haptic feedback has then been introduced for simulating perception and interaction with a highly complex 3D object: the user's self-avatar. We indeed introduced the notion of "Pseudo-Haptic Avatars" (Gomez, 2014) and showed how the visual animation of a self-avatar could be artificially modified in real-time in order to generate different haptic perceptions. In our experimental setup participants could watch their self-avatar in a virtual environment in mirror mode (see Figure 2D). 
They could map their gestures on the self-animated avatar in real-time using a Kinect. The experimental task consisted in a weight lifting with virtual dumbbells that participants could manipulate by means of a tangible stick. We tested three kinds of modification of the visual animation of the self-avatar: an amplification (or reduction) of the user motion, a change in the dynamic profile of the motion (temporal animation), or a change in the posture of the avatar (angle of inclination). Thus, to simulate the lifting of a "heavy" dumbbell, the avatar animation was distorted in real-time using: a decrease in user's visual motion, a slower dynamics, and a larger angle of inclination of the avatar. Experimental results showed that users were well able to discriminate weights using this pseudo-haptic feedback by relying only on the avatar motion and posture. This technique could for instance be used in applications such as sport training, exercise games, or industrial training.

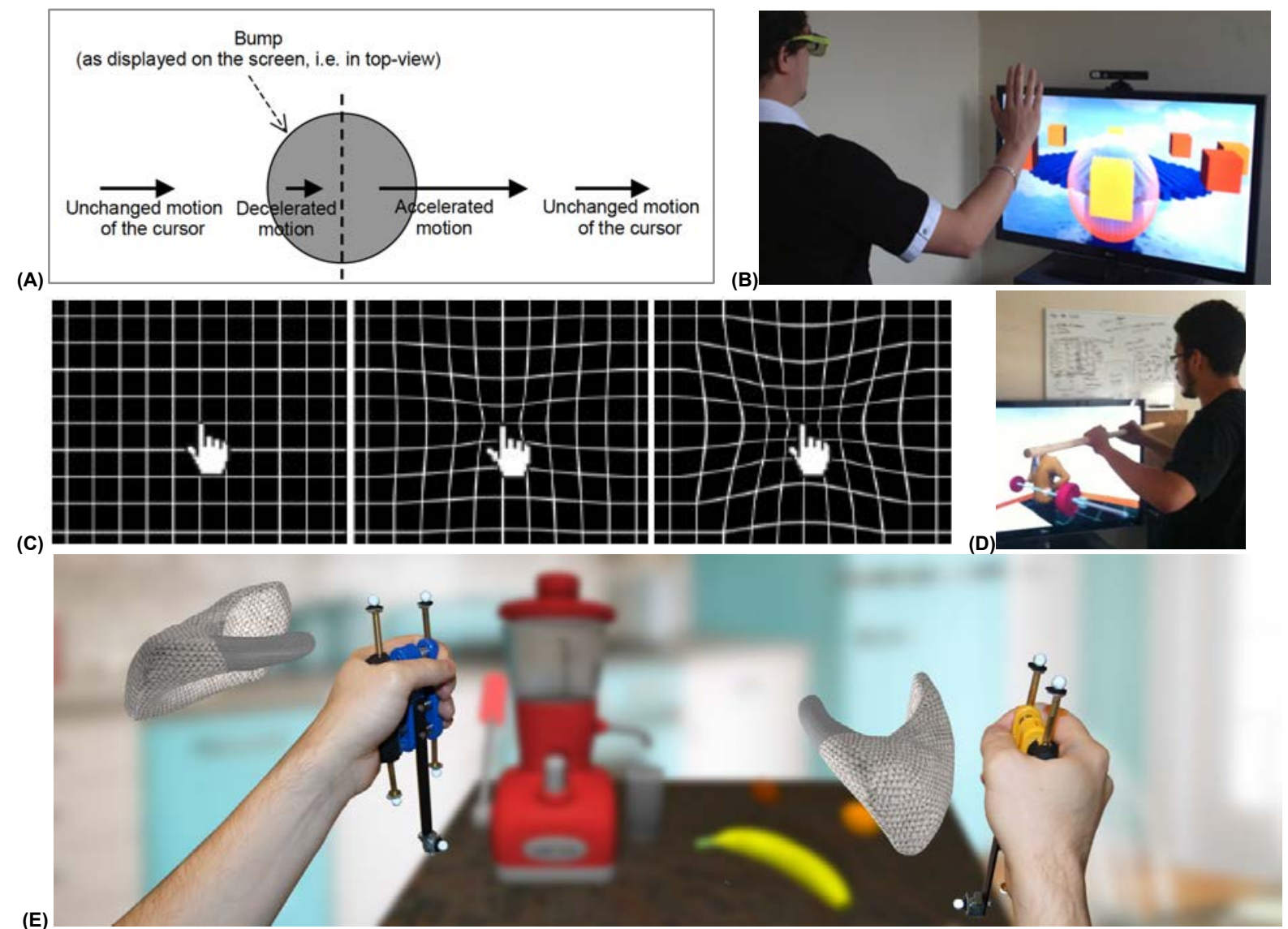

Figure 2: Pseudo-haptic effects. (A) Pseudo-haptic texture: simulating passing over a bump shape on screen by playing with the speed of the mouse cursor. (B) $3 D$ carousel: a carousel-like ring menu is rotated using swipe gestures, and is augmented with friction pseudo-haptic effects that can repulse or attract the user towards pre-determined items. (C) Elastic image: simulating the local elasticity of a $2 D$ image with a procedural deformation algorithm using the "pressure" corresponding to the time elapsed when clicking. (D) Pseudo-haptic avatar: the user can lift different virtual dumbbells using Kinect-based gestures recognition, and then perceive different virtual weights using pseudo-haptic effects applied on the self-avatar visual animation. (E) Virtual Mitten: an engineered hand-exerciser is used to grasp and manipulate virtual objects via a mitten metaphor augmented with pseudo-haptic effects enabling to feel different levels of grasping efforts.

All these examples illustrate how a spatiotemporal sensory conflict introduced and well controlled in the perception-action loop can produce a wide range of haptic sensations and improve 3D interaction. The visual motion is here distorted in a synchronized way with the user's physical motion or sensorymotor action. The resulting pseudo-haptic percept corresponds to the subjective reinterpretation of these stimuli, and to an optimal visuo-haptic perception of a world, which must remain coherent, depending on the interaction context. Interestingly a similar decrease in speed will be interpreted in one context as a texture effect and in another context as a change in mass. This suggests that many 
more pseudo-haptic effects could be invented, considering the great number of potential contexts and scenarios of VR applications.

\section{Self-motion illusions with the "haptic motion"}

A second series of results illustrate how introducing a haptic cue which is, this time, well consistent and synchronized with visual feedback can be used to improve self-motion sensations in VR.

The sensation of self-motion is intrinsically a multimodal perception involving many sensory channels: visual, tactile, proprioceptive, vestibular, and even auditory. A good way to generate selfmotion sensation in VR consists in watching a visual motion on a large screen or wearing an HMD with a large field of view. The peripheral visual stimulation can then be sufficient to induce a "vection" illusion. Vection is a well-known illusion of self-motion that most people have already experienced, for instance, when seated in a standing train while another train starts to move aside. In this situation, people would feel that they move in the opposite direction of the produced optic flow although they are steady.

We first showed in two experiments (Ouarti, 2014) that the vection illusion induced by the visual feedback of a virtual environment could be strongly improved in presence of an additional haptic force cue applied in the hands of the user. This force must be proportional to the acceleration present in the optical flow (Figure 3A). We strapped the shoulders of participants to ensure that the effect was not related to vestibular or proprioceptive feedback. And we found that the presence of this redundant haptic information had a significant and positive effect on the occurrences of the vection illusion, but also on its duration, onset, and on the perceived intensity of the sensation of self-motion.

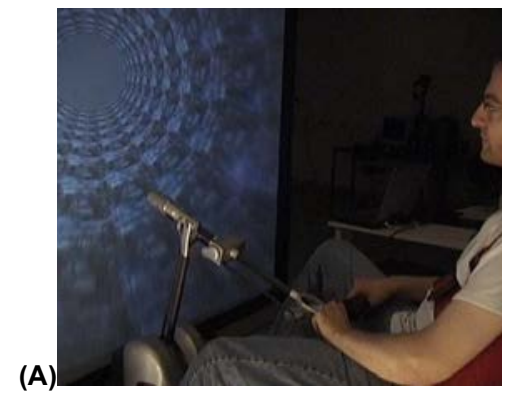

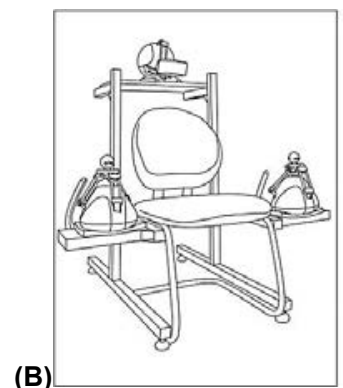

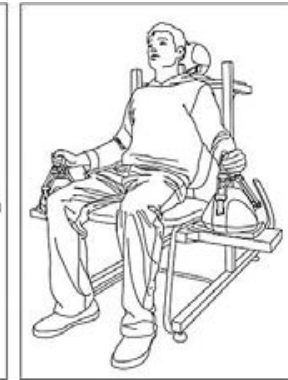

(C)

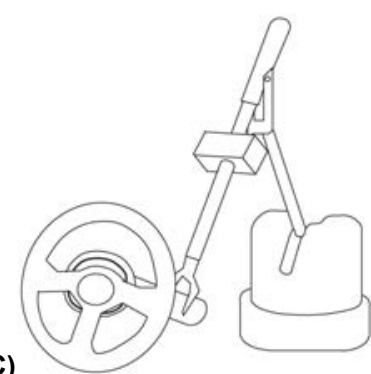

Figure 3: Haptic Motion. (A) Experimental apparatus used in (Ouarti, 2014), (B) HapSeat device, and (C) concept of driving simulator based on haptic motion.

One advantage of this technique, that we called "Haptic Motion", compare to the use of a classical motion platform, is the possibility to generate a sensation of acceleration during a very long time, and in any 3D direction or orientation. Thus, we can foresee a wide range of usages, notably in the entertainment industry, but also, more generally, in VR applications relying on motion or navigation in virtual worlds.

One application of this approach is the HapSeat device (see Figure 3B), which enables to produce motion sensations in a consumer living-space (Danieau, 2012). Instead of moving the entire user body as it is traditionally done with motion platforms, we proposed to stimulate only several specific parts of the body. The approach consists in applying multiple force-feedback so to generate a complete (6 degrees-of-freedom) sensation of self-motion, while being seated and experiencing a passive VR navigation. In our prototype, we used three low-cost haptic actuators arranged around the seat, in the two armrests and the headrest. A user study was conducted and participants reported that using the HapSeat increased significantly the quality of experience when watching virtual navigations. A second application is the design of a new kind of driving simulator (see Figure 3C). In this case, the motion platform would be substituted by the use of an actuated driving wheel, able to exert forces at the level of the hands of the driver. Following our haptic motion approach, these forces could simulate the 
motion felt by the user inside the vehicle such as when braking or accelerating (front/back longitudinal forces) or when turning (lateral forces).

\section{Body-ownership illusions using visuo-tactile stimulations}

Another approach shows how to exploit a synchronized stimulation between vision and touch together with a spatial discrepancy (offset) between them, in order to generate powerful body-ownership illusions in virtual reality.

The "body-ownership sensation" is classically depicted as the feeling of owning one's body and experiencing that this body is the source of sensations. It is currently of great interest for the VR community to better understand how and to what extent it is possible to develop a sense of ownership in virtual environments towards more or less realistic self-avatars.

A famous example of body-ownership illusion is the Rubber-Hand Illusion (RHI) originally described by Botvinick and Cohen. In their setup, the participant was seated with the left arm resting upon a table. A standing screen was positioned to hide the arm from participant's view. A life-sized rubber model of hand was placed on the table in front of the participant who was explicitly asked to keep eyes fixed on the rubber hand. Two small paintbrushes were manipulated by an experimenter to stroke simultaneously both the real hand and the rubber hand, synchronizing the timing of the two brushings as closely as possible. After ten minutes of such visuo-haptic stimulation, it was found that the participant "feel as if the rubber hand had sensed the touch". In other words, an illusion of ownership is observed towards the artificial hand. Later on, follow-up studies showed that the RHI can be obtained in VR, and also stressed the importance of the synchronization between the visual and haptic brushing feedback. A temporal delay introduced between the two sensory stimulations is found to break the illusion.

In a recent study (Hoyet, 2016), we could show how to generate body-ownership illusions when being immersed with a virtual body that is structurally different from ours. We considered the peculiar case of having a virtual body with a 6-digit hand (see Figure 4A). We got inspired from the RHI conditioning method to foster the appropriation of the 6-finger hand and generate a "6-Finger Illusion" (6FI). Participants controlled the six-digit hand from a first-person view while wearing an HMD. They performed two tasks successively. In the first task, participants manipulated the virtual hand while mimicking finger movements presented in the virtual scene. The second task was directly inspired by the RHI experiment: an experimenter stroked the participants' real fingers with a brush while a synchronous virtual brushing was presented in the virtual scene (see Figure 4B). In the case when the virtual brush was stroking the sixth digit, the real ring finger was synchronously stroked so to provide a consistent tactile stimulation. We measured how participants behaved when asked to lift every virtual digit. Interestingly, when the additional (sixth) digit was pointed, participants lifted indeed a real finger (ring or pinky) in $96 \%$ of cases. Our questionnaires results support that participants responded very positively to the possibility of controlling the six-digit hand despite the structural difference, and accepted to some extent the virtual hand and all the individual digits as their own. 

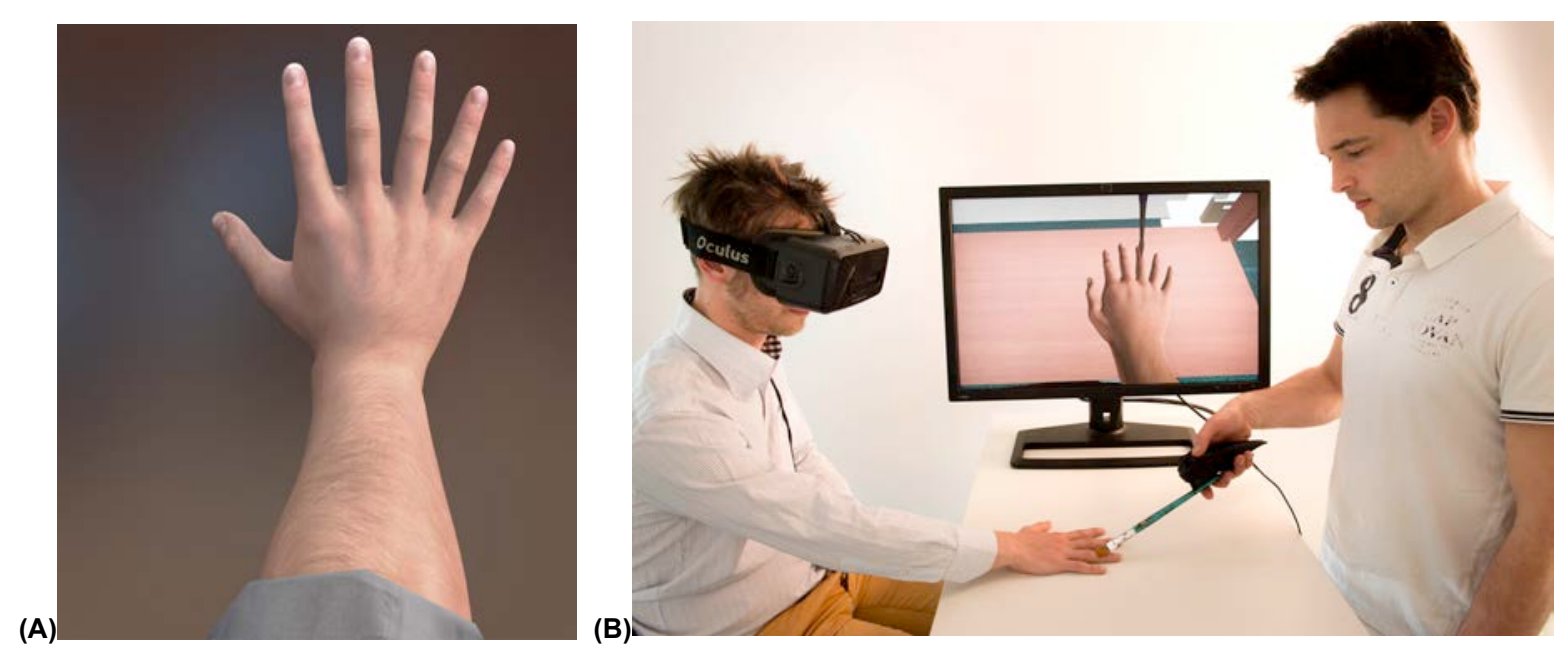

Figure 4: The 6-Finger Illusion (Hoyet, 2016). (A) 3D model of the 6-digit hand used in our experiment. (B) Experimental procedure for visuo-tactile stimulation.

Such conditioning protocol relying on synchronized visuo-tactile stimulations shows great potential for augmenting virtual embodiment in VR. Adding haptic cues synchronized with visual ones seems indeed to increase strongly the belief of participants, and could be encouraged when targeting applications in which the virtual body of the user plays an important role. In addition to videogames and immersive entertainment, we can think of numerous applications such as vocational or sport training, but also cybertherapy, or motor re-education.

\section{Conclusion}

When considering the combination of vision and touch in virtual environments we believe that "one plus one equals three". By playing carefully with two distinct sources of stimulation it becomes possible to create alternate and powerful perceptual experiences in VR. In our first example, introducing a spatiotemporal conflict and distorting visual motions can generate various haptic sensations and pseudo-haptic effects. In our second example, adding a force cue well synchronized with the visual stimulation can induce powerful self-motion sensations. In our third example, a tactile stimulation presented synchronously with visual feedback but with a spatial offset, enables to map the tactile sensations onto a fake virtual limb and generate body-ownership illusion.

Of course, several open questions remain related to the reconstructed percepts coming from these artificial situations. In particular, how do they differ from real-life situations? What do they reveal regarding human perception? Do they influence perception in reality, especially after long exposure in VR? Neuroimagery could help clarifying the cognitive processes involved, and eliciting the potential brain plasticity. In any case we need further works and fundamental studies to better understand and characterize multi-sensory integration in virtual environments.

The best is probably to come regarding the exploitation of vision and touch and, more generally, multi-sensory feedback in VR. We have addressed a subset of sensory combinations, and many more remain to be tested. We thus invite the VR community to further enter this playground and "play with senses" in virtual environments.

\section{Acknowledgements}

The author is deeply grateful to his colleagues and collaborators who have contributed to the results presented in this article: Merwan Achibet, Ferran Argelaguet, Alain Berthoz, Marc Christie, Marco Congedo, Fabien Danieau, Julien Fleureau, Pierre Gaucher, Edouard Gentaz, David Gomez, Philippe Guillotel, Ludovic Hoyet, Maud Marchal, Nicolas Mollet, Nizar Ouarti, and Jerome Royan. 


\section{Author}

Anatole Lécuyer is senior researcher and head of Hybrid team at Inria, Rennes, France. Contact him at anatole.lecuyer@inria.fr.

\section{References}

1. M. Achibet et al., "The Virtual Mitten: A novel interaction paradigm for visuo-haptic manipulation of objects using grip force", Proc. of IEEE 3DUI, 2014

2. F. Argelaguet et al., "Elastic Images: Perceiving Local Elasticity of Images through a Novel Pseudo-Haptic Deformation Effect", ACM TAP, 10:3, 2013

3. A. Berthoz, "The Brain's Sense of Movement", Harvard University Press, 2002

4. M. Congedo, A. Lécuyer, and E. Gentaz, "The Influence of Spatial De-location on Perceptual Integration of Vision and Touch", Presence, 15: 3, 2006

5. V. Cornilleau-Pérès and J. Droulez, "Application of the Coherence Scheme to the Multisensory Fusion Problem", Multisensory Control Movement, A. Berthoz (Ed), Oxford University Press, 1993

6. F. Danieau et al., "HapSeat: Producing Motion Sensation with Multiple Force-feedback Devices Embedded in a Seat", Proc. of ACM VRST, 2012

7. M.O. Ernst and M.S. Banks, "Humans Integrate Visual and Haptic Information in a Statistically Optimal Fashion", Nature, 415, 429-433, 2002

8. P. Gaucher et al., "A novel 3D Carousel based on Pseudo-Haptic Feedback and Gestural Interaction for Virtual Showcasing", Proc. of IEEE 3DUI, 2013

9. D.A. Gómez Jáuregui et al., "Toward "Pseudo-Haptic Avatars": Modifying the Visual Animation of Self-Avatar Can Simulate the Perception of Weight Lifting", IEEE TVCG, 2014

10.L. Hoyet et al., "Wow! I Have Six Fingers!: Would You Accept Structural Changes of Your Hand in VR?", Frontiers in Robotics and AI, 3:27, doi: 10.3389/frobt.2016.0002, 2016

11.A. Lécuyer, "Simulating Haptic Feedback using Vision: a Survey of Research and Applications of Pseudo-Haptic Feedback", Presence, 18:1, 39-53, 2009

12.M. Meehan, B. Insko, M. Whitton, and F.P. Brooks, "Physiological measures of presence in stressful virtual environments". Proc. of ACM SIGGRAPH, 2002

13.N. Ouarti, A. Lécuyer and A. Berthoz, "Haptic Motion: Improving Sensation of Self-Motion in Virtual Worlds with Force Feedback", Proc. of IEEE HAPTICS, 2014

14.I. Rock and J. Victor, "Vision and touch: An experimentally created conflict between the two senses", Science, 143, 594-596, 1964

15. M. Slater, A. Steed, “A virtual presence counter”, Presence, 9:5, 413-434, 2000 